\title{
Resection of cerebral gangliogliomas causing drug-resistant epilepsy: short- and long-term outcomes using intraoperative MRI and neuronavigation
}

\author{
Björn Sommer, MD,, Cornelia Wimmer, ${ }^{1}$ Roland Coras, MD, ${ }^{2}$ Ingmar Blumcke, MD, ${ }^{2}$ \\ Bogdan Lorber, MD, MSc, ${ }^{4}$ Hajo M. Hamer, MD, ${ }^{3}$ Hermann Stefan, MD, PhD, ${ }^{3}$ \\ Michael Buchfelder, MD, PhD, ${ }^{1}$ and Karl Roessler, MD, PhD' \\ Departments of ${ }^{1}$ Neurosurgery and ${ }^{2}$ Neuropathology, and ${ }^{3}$ Epilepsy Center, Department of Neurology, University Hospital \\ Erlangen, Germany; and ${ }^{2}$ Department of Neurology, University Medical Center Ljubljana, Slovenia
}

\begin{abstract}
OBJECT Cerebral gangliogliomas (GGs) are highly associated with intractable epilepsy. Incomplete resection due to proximity to eloquent brain regions or misinterpretation of the resection amount is a strong negative predictor for local tumor recurrence and persisting seizures. A potential method for dealing with this obstacle could be the application of intraoperative high-field MRI (iopMRI) combined with neuronavigation.

METHODS Sixty-nine patients (31 female, 38 male; median age $28.5 \pm 15.4$ years) suffering from cerebral GGs were included in this retrospective study. Five patients received surgery twice in the observation period. In 48 of the $69 \mathrm{pa}-$ tients, 1.5-T iopMRI combined with neuronavigational guidance was used. Lesions close to eloquent brain areas were resected with the implementation of preoperative diffusion tensor imaging tractography and blood oxygenation level-dependent functional MRI (15 patients).

RESULTS Overall, complete resection was accomplished in 60 of 69 surgical procedures (87\%). Two patients underwent biopsy only, and in 7 patients, subtotal resection was accomplished because of proximity to critical brain areas. Excluding the 2 biopsies, complete resection using neuronavigation/iopMRI was documented in 33 of 46 cases (72\%) by intraoperative imaging. Remnant tumor mass was identified intraoperatively in 13 of 46 patients (28\%). After intraoperative second-look surgery, the authors improved the total resection rate by 9 patients (up to $91 \%$ [ 42 of 46 ]). Of 21 patients undergoing conventional surgery, $14(67 \%)$ had complete resection without the use of iopMRI. Regarding epilepsy outcome, 42 of 60 patients with seizures (70\%) became completely seizure free (Engel Class IA) after a median follow-up time of $55.5 \pm 36.2$ months. Neurological deficits were found temporarily in $1(1.4 \%)$ patient and permanently in $4(5.8 \%)$ patients.
\end{abstract}

CONCLUSIONS Using iopMRI combined with neuronavigation in cerebral GG surgery, the authors raised the rate of complete resection in this series by $19 \%$. Given the fact that total resection is a strong predictor of long-term seizure control, this technique may contribute to improved seizure outcome and reduced neurological morbidity.

http://thejns.org/doi/abs/10.3171/2014.10.FOCUS14616

KEY WORDS intraoperative high-field MRI; surgery; tumor-related epilepsy; surgical complications; seizure control

$\mathrm{P}$ RIMARY low-grade brain tumors such as astrocytomas, dysembryoplastic neuroepithelial tumors, or gangliogliomas (GGs) are often associated with epilepsy. ${ }^{19,26,27,32}$ GGs are especially responsible for a large proportion of patients with drug-resistant seizures, particularly when located in the temporal lobe. ${ }^{17,30}$ With $60 \%-$ $95 \%$ of patients presenting with seizures as a primary symptom, between $20 \%$ and $40 \%$ develop drug-resistant epilepsy. ${ }^{24,32}$

Complete resection of tumorous lesions associated with epilepsy remains the major goal of surgery, which is associated with freedom from seizures ${ }^{7,15}$ and prolonged overall and progression-free survival in high percentages. ${ }^{16}$ Although GGs are considered "benign" tumors, recurrenc-

ABBREVIATIONS AED = antiepileptic drug; ECoG = electrocorticography; GG = ganglioglioma; GTR = gross-total resection; iopMRI = intraoperative high-field MRI; MEG = magnetoencephalography.

SUBMITTED September 1, 2014. ACCEPTED October 20, 2014.

INCLUDE WHEN CITING DOI: 10.3171/2014.10.FOCUS14616.

DISCLOSURE The authors report no conflict of interest concerning the materials or methods used in this study or the findings specified in this paper. 
es of WHO Grade I GGs are reported for approximately $1 \%$ of patients; recurrence rates increase to $25 \%-33 \%$ for atypical WHO Grade II tumors and to $38 \%-60 \%$ for WHO Grade III tumors. Atypical transformation into a WHO Grade II or III tumor is seen in $9.2 \%-10.3 \%$ and $2.5 \%-5.2 \%$ of patients, respectively. ${ }^{2,15,16}$ One reason for recurrence is incomplete resection after lesionectomy. Moreover, malignant transformation, and thus poor tumor control, has been associated with incomplete resections in the past. ${ }^{10}$ Other factors associated with unsatisfactory seizure and clinical outcomes are atypical or even high-grade GGs (WHO Grades II and III), lesions located in the vicinity of or within eloquent brain areas leading to subtotal lesionectomy, and duration of epilepsy longer than 1 year. ${ }^{6,16}$

The use of intraoperative high-field MRI (iopMRI) and neuronavigation might contribute to improvement in the extent of resection, seizure control, and neurological outcome in patients suffering from lesional drug-resistant epilepsy. ${ }^{18,21,22}$ In this study, we examined 69 adult patients suffering from GGs who underwent surgery in our hospital to determine predictive factors for seizure control, tumor recurrence, and neurological deficits over a shortterm and a long-term period.

\section{Methods \\ Patient Population}

From a total of 402 consecutive patients who underwent surgery for drug-resistant epilepsy at our department between December 2002 and April 2013, we performed 74 surgical procedures in 69 patients in whom the histopathological diagnosis was WHO Grade I, II, or III GG (Table 1). We analyzed these cases retrospectively by screening the patients' charts, surgical documents, and MR images. In addition to demographic data, included items were surgery with or without the use of iopMRI and neuronavigation, extent of resection (gross-total resection [GTR], subtotal resection, biopsy), tumor volume, residual tumor evaluated on long-term postoperative MRI, tumor recurrence, presence of drug-resistant epilepsy or sporadic seizures, number of antiepileptic drugs (AEDs) taken before and after the operation, postoperative neurological deficits, seizure outcome, recurrence of tumor, and reoperation.

Written informed consent was obtained from each patient. The local ethics committee approved the use of iopMRI.

Of 69 patients, 51 met the criteria for drug-resistant epilepsy according to the proposal by the International League Against Epilepsy (ILAE). ${ }^{14}$ Furthermore, we retrospectively classified the seizure type according to the recent ILAE proposal. ${ }^{1}$ All these patients underwent an extensive presurgical investigation according to the epilepsy protocol of the Center of Epilepsy, University Hospital Erlangen, which is described in detail elsewhere.,21,23 Additional magnetoencephalography (MEG) was performed in 9 patients using a 74-channel two-sensor system (Magnes II or whole-head MEG system; 4-D Neuroimaging). Nine patients presented with first-time sporadic seizures and received scalp electroencephalography and 1.5-T MRI. In the remaining 9 patients, no history of seizures was present.

\section{Data Acquisition and Analysis}

Localization of Eloquent Brain Areas and Fiber Tracts

We identified the Broca and Wernicke centers and the motor cortex (hand and foot areas) in 10 patients using functional MRI and blood oxygenation level-dependent imaging with stimulation paradigms as described previously. ${ }^{8,21}$ All functional MRI protocols consisted of a localizer scan (scanning time 6 seconds), high-resolution echo-planar imaging (25 continuous slices of 3-mm thickness, TE $60 \mathrm{msec}$, TR $2470 \mathrm{msec}$, image matrix 64 $\times 64$, FOV $240 \mathrm{~mm}$ ), and T1-weighted 3D magnetizationprepared rapid-acquisition gradient-echo sequences (for language area localization: slice thickness $1 \mathrm{~mm}$, TE 60 msec, TR $2470 \mathrm{msec}$, image matrix $256 \times 256$, FOV 192 $\mathrm{mm}$, scanning time 7 minutes 30 seconds; for motor area localization: slice thickness $1 \mathrm{~mm}$, TE $60 \mathrm{msec}$, TR 1580 msec, image matrix $256 \times 256$, FOV $192 \mathrm{~mm}$, scanning time 3 minutes 13 seconds). Imaging was conducted on a 1.5-T MR clinical whole-body scanner (Magnetom Sonata, Siemens Medical Solutions).

In 15 patients major white matter tracts such as pyramidal, visual, or language tracts were detected using the diffusion tensor imaging technique. In short, water diffusion in brain tissue is directed by the anatomical structures, such as the white matter fibers. Applying special MRI sequences enables the calculation and 3D visualization of the fiber tracts, which is also known as "tractography." In our setting, we applied a single-shot spin-echo diffusionweighted echo-planar imaging sequence (slice thickness $1.9 \mathrm{~mm}$, TE $86 \mathrm{msec}$, TR $9200 \mathrm{msec}$, image matrix 128 $\times 128$, FOV $240 \mathrm{~mm}$ ). The optic radiation, pyramidal tract, and the arcuate fascicle were reconstructed with this method as described previously. ${ }^{21}$

To calculate the minimal distance between eloquent brain areas, fiber tracts, and the GG, we used the "ruler" function of the iPlan software and scanned the proper MRI slices in every axis.

\section{Intraoperative Electrocorticography}

In 12 patients, intraoperative electrocorticography $(\mathrm{ECoG})$ for guidance of resection and identification of the epileptogenic zone was used. We applied one or a combination of up to four 4- or 6-contact subdural strips, 4-contact depth electrodes for hippocampal and amygdala positioning, or 32-electrode subdural grids (Ad-Tech Medical Instrument Corporation and PMT/Permark Corporation). The cortex exposed through craniotomy was monitored by an electrode rack consisting of 16 surface electrodes. ${ }^{17}$ The correct anatomical positions of the electrodes were confirmed by iopMRI. In all 12 patients, a distinct epileptogenic area was defined from the ECoG measurements according to interictal spike activity, leading to "tailored" resection. In each patient, this area corresponded to the resulting resection volume that we segmented using the neuronavigation software.

\section{Definition of the Extent of Resection}

Using the iPlan 2.6 neuronavigation software (Brainlab AG), we screened the appropriate initial intraoperative T2-weighted MR images and segmented each lesion man- 
TABLE 1. Demographic data of the study population*

\begin{tabular}{|c|c|c|c|c|c|}
\hline Variable (no. of patients) & $\begin{array}{l}\text { No. of Patients w/ } \\
\text { Seizures }(n=60)\end{array}$ & Engel Class la & Engel Class | \& II & Engel Class III or IV & p Value \\
\hline \multicolumn{6}{|l|}{ Sex } \\
\hline Male (38) & 29 & $72(21 / 29)$ & $97(28 / 29)$ & $3(1 / 29)$ & $0.782 \dagger$ \\
\hline Female (31) & 31 & $68(21 / 31)$ & $84(26 / 31)$ & $16(5 / 31)$ & \\
\hline Median age at seizure onset (yrs) & $16.0 \pm 15.2$ & & & & $0.02 \ddagger$ \\
\hline Median age at surgery (yrs) & $30.5 \pm 14.7$ & & & & $0.05 \ddagger$ \\
\hline \multicolumn{6}{|l|}{ Lesion location } \\
\hline Rt hemisphere (45) & 36 & $83(30 / 36)$ & $94(34 / 36)$ & $6(2 / 36)$ & $0.023 \dagger$ \\
\hline Lt hemisphere (24) & 24 & $50(12 / 24)$ & $83(20 / 24)$ & $17(4 / 24)$ & \\
\hline Temporomesial (32) & 32 & $59(19 / 32)$ & $88(28 / 32)$ & $12(4 / 32)$ & $0.011 \dagger$ \\
\hline Extratemporomesial (37) & 28 & $86(24 / 28)$ & $93(26 / 28)$ & $7(2 / 28)$ & \\
\hline Eloquent brain region (15) & 14 & $79(11 / 14)$ & $93(13 / 14)$ & $7(1 / 14)$ & $0.520 \dagger$ \\
\hline Noneloquent brain region (54) & 46 & $63(29 / 46)$ & $87(40 / 46)$ & $13(6 / 46)$ & \\
\hline \multicolumn{6}{|l|}{ Epilepsy } \\
\hline \multicolumn{6}{|l|}{ No seizures (9) } \\
\hline \multicolumn{6}{|l|}{ Duration of epilepsy (yrs) } \\
\hline Drug resistant (51) & $14 \pm 11.7$ & $67(34 / 51)$ & $88(45 / 51)$ & $12(6 / 51)$ & $0.469 \ddagger$ \\
\hline Sporadic seizures (9) & $0.3 \pm 4.4$ & $89(8 / 9)$ & $11(1 / 9)$ & $0(0 / 9)$ & $0.651 \ddagger$ \\
\hline \multicolumn{6}{|l|}{ Type of seizures } \\
\hline Focal w/o impairment of consciousness (40) & & $63(25 / 40)$ & $93(37 / 40)$ & $7(3 / 40)$ & $0.084 \dagger$ \\
\hline Focal w/ impairment of consciousness (55) & & $71(39 / 55)$ & $93(51 / 55)$ & $7(4 / 55)$ & $0.631 \dagger$ \\
\hline Aura (45) & & $64(29 / 45)$ & $91(41 / 45)$ & $9(4 / 45)$ & $0.192 \dagger$ \\
\hline Evolving to a bilat convulsive seizure (47) & & $72(34 / 47)$ & $89(42 / 47)$ & $11(5 / 47)$ & $0.504 \dagger$ \\
\hline
\end{tabular}

* The total number of patients was 69 . Results are medians \pm 1 SD or percentage (no./total no.).

$\dagger$ Two-tailed Fisher exact analysis.

$\ddagger$ One-way ANOVA.

ually in every axis using the ruler function of the software. The resulting tumor volume was calculated by the software and was defined as the maximum tumor mass that could be achieved for GTR.

We defined subtotal resection as a volume reduction of 90\%-99\% and GTR as complete removal of the segmented tumor mass. In cases of re-resection, remnant tumor volume was measured by comparing the corresponding initial preoperative and intraoperative MR images, and a new outline of the remaining tumor contours was obtained using our neuronavigation software.

\section{Surgical Workflow}

Details of the intraoperative workflow and setup have been published before. ${ }^{13,21}$ In summary, imaging started after inducing general anesthesia and rotating the patient into the iopMRI scanner (1.5-T MRI, Magnetom Sonata Maestro Class, Siemens Medical Solutions). The intraoperative sequences were acquired as described previously. ${ }^{21,22}$ Overall, the mean intraoperative scanning time was 13.9 minutes. The data set was fused with preoperative functional MRI data using our neuronavigation software (iPlan 2.6, Brainlab AG). We used the T2-weighted images for segmentation of the lesion and coregistration of functional data (Fig. 1). After planning the ideal trajectory, we transferred the navigation plan to the surgical microscope (OPMI Pentero, Zeiss). Coregistration of preopera- tive and initial intraoperative MRI sequences with anatomical structures was performed with a median error of $1.4 \pm 0.7 \mathrm{~mm}$ of the navigation system.

\section{Surgical Technique}

With the aid of navigational trajectory and superimposing the boundaries of the segmented lesion, the skin flap and craniotomy were planned before the skin incision. Lesionectomy was performed using the microsurgical technique. If the neurosurgeon had the impression that the intended resection extent was reached and/or he was too close to the displayed boundaries of eloquent brain areas or white matter fiber tracts, an intraoperative control MR scan was acquired. In cases of resectable residual lesion, surgery was continued, and completeness of the lesionectomy was documented in the final iopMRI before the closing procedure. In cases in which the residual tumor mass was considered impossible for resection because of its proximity to eloquent brain regions, we performed the standard dural closure and reinsertion of the skull bone right away.

\section{Definition of Postoperative Neurological Deficits}

Clinical investigation of neurological deficits was performed before surgery, on the day of discharge, 3 months after surgery, and, most recently, for completing the follow-up data for this study. If a patient suffered from 


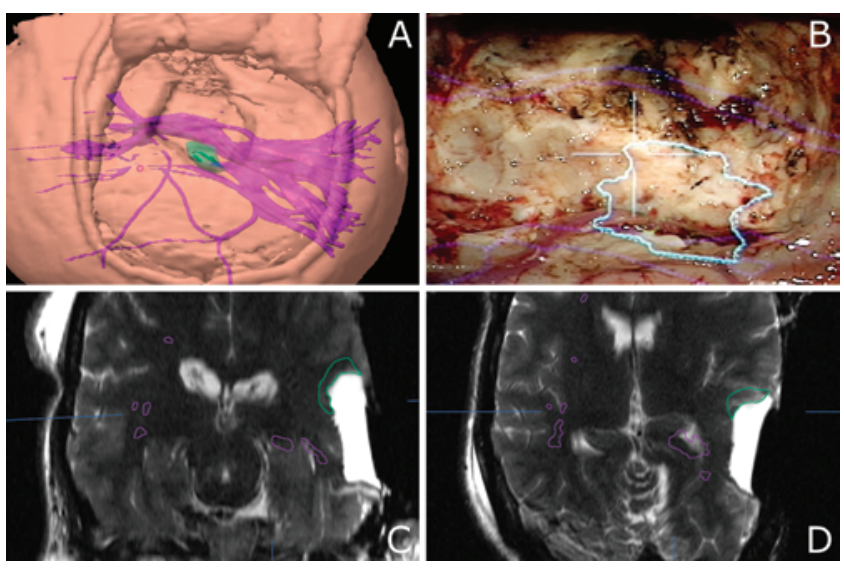

FIG. 1. Intraoperative data of Patient 37 with a temporal GG. A: 3D reconstruction of T1-weighted 3D magnetization-prepared rapid-acquisition gradient-echo sequences depicting the skin flap and craniotomy. On the cranial corner of the initial resection cavity, the remnant tumor mass as determined by T2-weighted iopMRI in coronal (C) and axial (D) sequences is segmented in green. Update of functional neuronavigation data included reconstruction of the visual fiber tracts (purple). B: Microscopic view of the resection cavity, the projected segmented lesion (straight and dashed light-blue lines), and the course of the visual fiber tract (purple dotted lines) are shown.

postoperative complications that caused only minor limitations such as upper visual field deficits, discrete aphasia, or latent monoparesis, we considered that patient to have a "mild neurological deficit." Manifest hemiparesis, complete hemianopia, or global aphasia leading to a dependency on further care or immobility was referred to as a "severe neurological deficit."

\section{Definition of Seizure Outcome}

The most recent Engel classification system was applied to evaluate postsurgery seizure outcome. ${ }^{5}$ Engel Classes Ia, I and II, and III and IV were defined as excellent, favorable, and poor outcomes, respectively (Tables 1 and 2). The patients' neurological and seizure outcome data were obtained by follow-up examinations in the University Hospital Erlangen Epilepsy Center in the outpatient department and via telephone interviews.

\section{Statistical Analysis}

Statistics were performed to identify correlations between dependent variables and seizure control. Potential confounding variables were the use of neuronavigation/ iopMRI, eloquent or noneloquent localization of the lesion, and drug-resistant and sporadic seizure occurrence resulting from the unbalanced sample sizes. Continuous variables are presented as medians with $1 \mathrm{SD}$ and included age at surgery, age at seizure onset, duration of epilepsy, preoperative tumor volume, and residual tumor volume. These variables were assessed by repeated-measures 2-way analysis of variance (ANOVA). To meet the requirements for a normal distribution, we examined each variable independently with a Shapiro-Wilk test. For homogeneity of variances, a Levene test was used. Bonferroni posthoc adjustment for multiple comparisons was used when differences displayed statistical significance.
Categorical variables were analyzed using the Fisher exact test. Seizure outcomes were dichotomized as excellent seizure control (Engel Class IA) or persistent seizures (Engel Class IB-IV). The SPSS statistical software package 21.0 for Windows (SPSS Inc., Chicago, Illinois) was used for statistical analysis. Significance was accepted when the $\mathrm{p}$ value was less than 0.05 .

\section{Results}

\section{Patients, Symptoms, and Surgeries}

For detailed demographic descriptions, see Table 1. The predominant seizure type was focal seizures with impairment of consciousness $(92 \%)$, and seizure frequency ranged from 80 per day to 1 every 2 years. Of the 69 patients (31 female and 38 male, median age $28.5 \pm 15.4$ years) with tumor-associated epilepsy and GGs, 5 patients underwent surgery twice during the observation period. Of 48 patients who underwent surgery with iopMRI and neuronavigation, 1 patient with a thalamic GG and 1 patient with the lesion located in the central sulcus underwent biopsy only (Table 2). Twenty-one patients underwent surgery without the use of neuronavigation and iopMRI. Although the proportion of patients with excellent seizure control was higher in the neuronavigation/iopMRI group (73\% vs 58\%, respectively), statistical analysis revealed no significant differences between the 2 groups $(\mathrm{p}=0.48)$.

Functional neuronavigation was applied in 15 patients with lesions located near eloquent brain areas (Table 1). The minimal mean distance between eloquent brain structures and the tumor was $1.6 \mathrm{~mm}$ (range $0-10 \mathrm{~mm}$ ).

\section{Extent of Resection}

Of 69 patients, complete resection was confirmed by late MRI 3 months after surgery in 60 cases (87\%). GTR was a strong significant predictor of excellent seizure control $(\mathrm{p}=0.007)$. Three of 5 patients with a WHO Grade I GG underwent a second surgery on recurrences despite the use of iopMRI and neuronavigation during the first surgery. The other 2 patients first had conventional surgery and then underwent surgery including neuronavigation/iopMRI at the time of the second surgery.

Multimodal navigation/iopMRI was used in a total of 48 patients, including in 2 biopsies performed because of the highly eloquent location of the lesion. In the remaining 46 cases, iopMRI confirmed complete resection in 33 patients $(72 \%)$. Remnant tumor mass was identified in 13 (28\%) of 46 patients by iopMRI (Fig. 2). In 4 of those patients, further resection was discontinued because of the tumor's proximity to eloquent brain areas and fiber tracts. After resegmentation of the residual tumor and update of neuronavigation (Fig. 1), the total resection rate was improved by 9 patients up to $91 \%$ (42 of 46), with 1 patient's tumor remaining incompletely resected (Table 2). It is interesting to note that 5 of those patients whose tumors were intraoperatively re-resected remained tumor free during the observational period and had excellent seizure control. In addition, the use of iopMRI for the detection of GTR was a strong significant predictor of freedom from seizures $(\mathrm{p}=0.001)$.

Of the 21 patients who underwent surgery without 
TABLE 2. Surgical data*

\begin{tabular}{|c|c|c|c|c|c|}
\hline Variable (no. of patients) & $\begin{array}{l}\text { No. of Patients w/ } \\
\text { Seizures }(n=60)\end{array}$ & Engel Class la & Engel Class I or II & Engel Class III or IV & $\mathrm{p}$ Value \\
\hline GTR $(60) \dagger$ & GTR (52)† & $77(40 / 52)$ & $90(47 / 52)$ & $10(5 / 52)$ & $0.007 \ddagger$ \\
\hline STR $(9) \dagger$ & STR (8)† & $25(2 / 8)$ & $75(6 / 8)$ & $25(2 / 8)$ & \\
\hline Type of surgery $(59) \S$ & & & & & NS $\ddagger$ \\
\hline Standard resection (temporal lobe) (11) & & $82(9 / 11)$ & $82(9 / 11)$ & $18(2 / 11)$ & \\
\hline Tailored resection (w/ ECoG) (12) & & $67(8 / 12)$ & $100(12 / 12)$ & $0(0 / 12)$ & \\
\hline Selective lesionectomy (17) & & $65(11 / 17)$ & $94(16 / 17)$ & $6(1 / 17)$ & \\
\hline Extended lesionectomy (19) & & $74(14 / 19)$ & $90(17 / 19)$ & $10(2 / 19)$ & \\
\hline \multicolumn{6}{|l|}{ Use of iopMRI \& neuronavigation } \\
\hline Yes (48) & 48 & $73(35 / 48)$ & $90(43 / 48)$ & $10(5 / 48)$ & $0.482 \ddagger$ \\
\hline No (21) & 12 & $58(7 / 12)$ & $83(10 / 12)$ & $17(2 / 12)$ & \\
\hline Patients w/ GTR after 1st iopMRI (33)ף & & $88(29 / 33)$ & $100(33 / 33)$ & $0(0 / 33)$ & $0.001 \ddagger$ \\
\hline Patients remaining STR after 1st iopMRI (4)ף & & $50(2 / 4)$ & $100(4 / 4)$ & $0(0 / 4)$ & NA \\
\hline Patients w/ GTR after 2nd iopMRI (9)ף & & $56(5 / 9)$ & $67(6 / 9)$ & $33(3 / 9)$ & $0.301 \ddagger$ \\
\hline Patients remaining STR after 2nd iopMRI (1)ף & & $100(1 / 1)$ & $0(0 / 1)$ & $0(0 / 1)$ & NA \\
\hline Biopsy (1) & & $0(0 / 0)$ & $0(0 / 1)$ & $1(1 / 1)$ & NA \\
\hline Tumor volume $\left(\mathrm{cm}^{3}\right)(46)^{\star *}$ & $7.2 \pm 14.0$ & $72(33 / 46)$ & $91(42 / 46)$ & $9(4 / 46)$ & $0.135 \dagger \dagger$ \\
\hline Residual tumor volume $\left(\mathrm{cm}^{3}\right)(12)^{\star *}$ & $1.6 \pm 2.5$ & $50(6 / 12)$ & $83(10 / 12)$ & $17(2 / 12)$ & $0.109 \dagger \dagger$ \\
\hline Histopathological results & & & & & NA \\
\hline WHO Grade I GG (64) & 57 & $79(45 / 57)$ & $88(50 / 57)$ & $12(7 / 57)$ & \\
\hline WHO Grade II GG (4) & 3 & $100(3 / 3)$ & $0(0 / 3)$ & $0(0 / 3)$ & \\
\hline WHO Grade III GG (1) & 0 & $0(0 / 0)$ & $0(0 / 1)$ & $0(0 / 1)$ & \\
\hline Cystic lesion (33) & & $61(20 / 33)$ & $94(31 / 33)$ & $6(2 / 33)$ & $0.493 \ddagger$ \\
\hline Calcified lesion (27) & & $70(19 / 27)$ & $100(27 / 27)$ & $0(0 / 27)$ & $0.531 \ddagger$ \\
\hline Cystic + calcified lesion (14) & & $64(9 / 14)$ & $86(12 / 14)$ & $14(2 / 14)$ & $1.000 \ddagger$ \\
\hline \multicolumn{6}{|c|}{$\begin{array}{l}\text { NA = not applicable; NS }=\text { no statistical significance; STR = subtotal resection. } \\
\text { * The total number of patients was } 69 \text {. Results are medians } \pm 1 \text { SD or percentage (no./total no.). } \\
\dagger \text { Resection as confirmed by latest follow-up MRI. } \\
\text { † Two-tailed Fisher exact test. } \\
\text { § Excluding } 1 \text { patient who underwent biopsy. } \\
\text { I Resection as confirmed by iopMRI. } \\
\text { ** As determined by iopMRI. } \\
\text { †† One-way ANOVA. }\end{array}$} \\
\hline
\end{tabular}

neuronavigation/iopMRI, 67\% (14 of 21) had no residual tumor mass or recurrence appear in late follow-up MRI. Late MRI follow-up scans were available for $66(95.6 \%)$ of 69 patients. The type of surgery, preoperative tumor volume, and morphological results of the GG had no impact on seizure outcomes (Table 2).

\section{Neurological Outcomes}

Transient neurological deficits were noted in 1 patient (1.4\%) who experienced a visual field deficit and loss of visual acuity, which resolved completely within weeks. Mild permanent neurological deficits occurred in $4.3 \%$ (3 of 69) of the cases; 1 patient had a mild Wernicke aphasia, and 2 patients suffered from contralateral upper visual field defects. A severe neurological deficit was seen in 1 patient (1.4\%) who suffered from a progressive global aphasia and spastic hemiparesis after biopsy of an atypical thalamic GG.

\section{Seizure Outcomes}

Altogether, excellent seizure control was achieved in
$70 \%$ (42 of 60) of the patients and favorable seizure outcome in $95 \%$ (57 of 60) of the patients suffering from epilepsy preoperatively (median follow-up time $55.5 \pm 36.2$ months). In patients with drug-resistant epilepsy, 67\% (34 of 51) were seizure free after surgery. The proportion of seizure-free patients taking AEDs was 41\% (21 of 51), and the proportion was $26 \%$ (13 of 51) for those whose seizures were Engel Class IA after discontinuation of AEDs. Patients with sporadic seizures were completely seizure free in 8 of 9 cases. In our statistical analysis, we found age at surgery and age at seizure onset to be predictive variables for seizure outcome. Younger patients (28.6 \pm 12.9 years) with earlier epilepsy onset $(17.3 \pm 13.3$ years $)$ were more likely to become seizure free than older patients $(45.7 \pm 12.4$ years; $\mathrm{p}=0.02)$ who developed seizures at a later age $(33.0 \pm 23.9$ years; $\mathrm{p}=0.05)$. The factors sex, eloquent or noneloquent localization, type of seizures, and duration of epilepsy were not associated with a statistically significant difference regarding seizure control.

The largest proportion of seizure-free patients with 

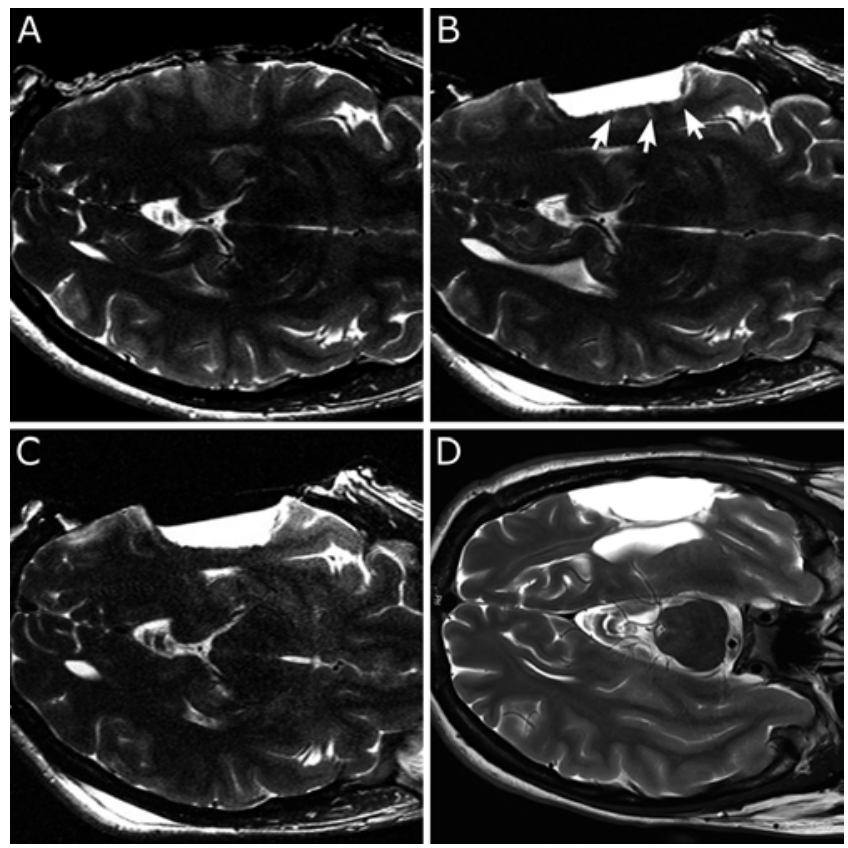

FIG. 2. A: Intraoperative axial T2-weighted MRI sequence of Patient 37. The neocortical temporal lesion is slightly blurry with a thickened cortical band. B: Intraoperative resection control with iopMRI shows remnant tissue on the mesial aspect of the resection border (white arrows). C: After intraoperative update of neuronavigation and resegmentation of remnant tumor tissue, complete tumor resection was accomplished. D: Follow-up image revealing no remnant tumor mass.

temporal GG was achieved when the lesion was located outside the temporomesial structures $(86 \%$ vs $59 \%$; $\mathrm{p}=$ 0.011). As an indirect effect of intraoperative re-resection of remnant tumor mass using neuronavigation/iopMRI, the number of patients with excellent seizure control was increased from $37(62 \%)$ of 60 to 42 (70\%) of 60 patients.

After 1 year, follow-up data were available from all the patients, and 46 of $60(77 \%)$ of them had excellent seizure control. At 2 and 4 years after surgery, seizure-free rates were $77 \%$ (41 of 53) and 74\% (28 of 38), respectively. Five years after surgery, 33 patients were available, with $79 \%$ (26 of 33) having excellent seizure control. Preoperative determination of the seizure focus using MEG was performed in 9 patients and intraoperative ECoG for tailored resection in 12 patients. However, neither one of those methods had a statistically significant effect on seizure outcome in this series (MEG $\mathrm{p}=0.23$, ECoG $\mathrm{p}=1.0$ ). Comparing the types of surgery, we also found no differences regarding seizure control (Table 2).

\section{General Surgical Complications}

No severe surgical complications such as intracranial hemorrhage, meningitis, or death occurred in this series. One (1.5\%) of 69 patients suffered from an iatrogenic pneumothorax after preoperative implantation of a central venous catheter in the subclavian vein. After a thoracic drain was placed for 3 days, the patient was brought to the general ward in stable condition. Left hemispheric brain edema occurred in another patient who presented with right-sided transient hemiparesis, which resolved completely under oral application of dexamethasone for 1 week.

\section{Postsurgical Follow-Up}

Overall, 2 (3.3\%) of 60 patients died during the observation period. One patient with an anaplastic GG died as a result of tumor progression despite combined radiochemotherapy. The other patient, with a WHO Grade I GG, died of malignant lung cancer.

\section{Discussion}

In this study, we analyzed the impact of the level of resection and the implication of iopMRI combined with neuronavigation during surgery of GGs in patients suffering mainly from focal epilepsy. We found a high rate of incompletely resected tumors during surgery by using iopMRI. Thus, our proposed surgical protocol included an immediate second-look surgery, which improved the resection rate. The improved resection rate even transformed to a higher percentage of seizure-free patients at the end of follow-up, compared with patients who underwent surgery without neuronavigation/iopMRI. In addition, we demonstrated that this approach leads to excellent long-term seizure outcome. To the best of our knowledge, this is the first published series on iopMRI and neuronavigation involving a large number of patients suffering from GG.

Because an optimal seizure outcome is associated mainly with complete resection in patients with GGs, ${ }^{6,15,16}$ a major goal of surgery is the identification and removal of the entire lesion. In 2002, Blümcke and Wiestler ${ }^{2}$ performed a retrospective analysis of epidemiological and clinical data from 326 patients with GGs. Incomplete resection was found in 12 (7.9\%) of 151 patients with a WHO Grade I GG. In addition, they observed local recurrence or malignant transformation in 3 of 12 atypical (WHO Grade II) and 3 of 8 anaplastic (WHO Grade III) GGs.

Especially in extratemporal lesions located close to eloquent brain areas, surgery is challenging, and even experienced surgeons tend to overestimate the extent of resection. ${ }^{3,25}$ Results of iopMRI in glioma, pituitary, and epilepsy surgery underline the benefits of intraoperative resection control. ${ }^{4,13,18,20}$ In combination with a neuronavigation system, these methods complement one another and increase the number of complete resections. In epilepsy-associated benign lesions such as GGs, use of this technique may decrease the instances of poor seizure control resulting from incomplete resection., ${ }^{3,11,22,28}$ In this analysis, complete resection was accomplished in $87 \%$ of the patients, as seen in late follow-up MR scans. Moreover, in $91 \%$ of the patients who underwent surgery including neuronavigation/ iopMRI, use of iopMRI determined complete resection. The positive confirmation of this early estimation of the resection extent, which certainly is vulnerable to internal measurement errors caused by, for example, intraoperative bleeding, insertion of a hemostyptic agent, or edema, was provided by late follow-up MR scans. Compared with total resection rates between $61 \%$ and $76 \%$ reported after "conventional" surgery without the use of intraoperative resection control, our results are very promising.,916,24,31

Although the patients who underwent surgery that included neuronavigation/iopMRI had lesions close to eloquent brain areas, and therefore had a higher risk of incomplete resection than those patients who underwent 
surgery without the multimodal approach, we achieved better results regarding excellent seizure control (73\% vs $58 \%$ Engel Class IA). Although GTR for the whole study population and GTR after the first intraoperative highfield MR scan for the subgroup using this multimodal approach were identified as one of the prognostic factors for freedom from seizures, one has to keep in mind the different sample sizes used for the statistical analysis (see "Study Limitations" below). Against this background, we conclude that our approach improves seizure control up to $88 \%$ (34 of 42 seizure-free patients with confirmed GTR as determined by first iopMRI scan). Compared with existing series, an Engel Class I outcome was reported in $63 \%-79 \%$ of patients undergoing epilepsy surgery. ${ }^{29,32}$ In a 2012 literature review by Southwell et al., ${ }^{24}$ the proportions of seizure-free adult patients with GGs were reported to be between $45 \%$ and $100 \%$. However, 13 of 19 of the listed studies consisted of small case series $(\leq 30)$. In the remaining series, $63 \%-86 \%$ of the patients remained seizure free, with a higher proportion of non-eloquent localization of GG and, consequently, a higher chance of GTR. Thus, our study clearly demonstrates the positive impact of iopMRI and neuronavigation guidance, with which excellent seizure control was reached in 13 cases despite the inclusion of GGs located in highly eloquent brain areas.

Against our expectations and contrary to results in previously published reports, ${ }^{6,15}$ patients with lesions located in the temporomesial region were less likely to achieve excellent seizure control than those with an extratemporal mesial location ( $59 \%$ vs $86 \%$, respectively). One reason could be the type of resection or resection extent. However, in our series, no statistically significant differences concerning these 2 factors were found. In our series, young patients whose seizures began early in their lives were more likely to become seizure free than were elderly patients who developed seizures at a later age $(p=0.02$ and 0.05 , respectively). These findings are consistent with those in the existing literature..$^{15,16}$

\section{Study Limitations}

The major limitations of this study are its retrospective nature and the lack of randomization in terms of patients undergoing surgery with or without neuronavigation/ iopMRI. Because of the unbalanced sample sizes favoring the use of iopMRI and neuronavigation versus conventional surgery, there is a selection bias accompanying our chosen methodological approach. Moreover, possible confounding variables are conceivable when comparing group effects. With this background, statistical differences should be interpreted carefully. In epilepsy surgery, the small number of cases and the tedious nature of drugresistant epilepsy make it extremely difficult to conduct well-powered randomized controlled trials. In our case, we have to justify refusing an available sophisticated, modern technology, such as multimodal neuronavigation, in order to have controls, which in our experience is accepted by few patients.

The accuracy of neuronavigation depends on the registration and errors, which may be the source of inadequate resection and postoperative neurological deficits. Shifting of brain tissue as a result of the loss of cerebrospinal fluid or simply the amount of tissue that is resected leads to a shifting of brain structures once it is taken away. ${ }^{12}$ We counter this problem by using iopMRI and updating neuronavigation data during the course of surgery. This technology, of course, leads to a prolonged operation time and requires more financial and human resources than does conventional surgery. Regarding the display of eloquent brain areas and white matter tracts, awake craniotomy with electrocortical stimulation is considered the gold standard for mapping those areas and defining critical brain structures. However, awake surgery and subcortical mapping entail a variety of problems, including the risk of severe anxiety during the procedure, pain during surgery, and generating epileptiform after-discharges with concomitant failure of awake cortical mapping.

\section{Conclusions}

To our knowledge, this is the first report on the application of neuronavigation and iopMRI for surgery in a large number of patients suffering from GG-associated epilepsy. Our results reveal a high rate of complete tumor resection with low neurological morbidity and mortality rates. Because GTR seems to be a key factor for tumor recurrence and seizure control, our data underline the value of intraoperative resection control resulting in a high number of seizure-free patients. In our opinion, the application of this sophisticated method is justified for improving overall outcomes in this rare patient group.

\section{References}

1. Berg AT, Berkovic SF, Brodie MJ, Buchhalter J, Cross JH, van Emde Boas W, et al: Revised terminology and concepts for organization of seizures and epilepsies: report of the ILAE Commission on Classification and Terminology, 20052009. Epilepsia 51:676-685, 2010

2. Blümcke I, Wiestler OD: Gangliogliomas: an intriguing tumor entity associated with focal epilepsies. J Neuropathol Exp Neurol 61:575-584, 2002

3. Buchfelder M, Fahlbusch R, Ganslandt O, Stefan H, Nimsky $\mathrm{C}$ : Use of intraoperative magnetic resonance imaging in tailored temporal lobe surgeries for epilepsy. Epilepsia 43:864873,2002

4. Buchfelder M, Schlaffer SM: Intraoperative magnetic resonance imaging during surgery for pituitary adenomas: pros and cons. Endocrine 42:483-495, 2012

5. Engel J, Van Ness PC, Rasmussen TB, Ojemann LM: Outcome with respect to epileptic seizures, in Engel J Jr (ed): Surgical Treatment of the Epilepsies. New York: Raven Press, 1993, pp 609-621

6. Englot DJ, Berger MS, Barbaro NM, Chang EF: Factors associated with seizure freedom in the surgical resection of glioneuronal tumors. Epilepsia 53:51-57, 2012

7. Englot DJ, Han SJ, Berger MS, Barbaro NM, Chang EF: Extent of surgical resection predicts seizure freedom in lowgrade temporal lobe brain tumors. Neurosurgery 70:921928,2012

8. Grummich P, Nimsky C, Pauli E, Buchfelder M, Ganslandt $\mathrm{O}$ : Combining fMRI and MEG increases the reliability of presurgical language localization: a clinical study on the difference between and congruence of both modalities. Neuroimage 32:1793-1803, 2006

9. Hu WH, Ge M, Zhang K, Meng FG, Zhang JG: Seizure outcome with surgical management of epileptogenic ganglioglioma: a study of 55 patients. Acta Neurochir (Wien) 154:855-861, 2012 
10. Im SH, Chung CK, Cho BK, Wang KC, Yu IK, Song IC, et al: Intracranial ganglioglioma: preoperative characteristics and oncologic outcome after surgery. J Neurooncol 59:173-183, 2002

11. Kelly JJ, Hader WJ, Myles ST, Sutherland GR: Epilepsy surgery with intraoperative MRI at 1.5 T. Neurosurg Clin $\mathbf{N}$ Am 16:173-183, 2005

12. Kuhnt D, Bauer MH, Nimsky C: Brain shift compensation and neurosurgical image fusion using intraoperative MRI: current status and future challenges. Crit Rev Biomed Eng 40:175-185, 2012

13. Kuhnt D, Ganslandt O, Schlaffer SM, Buchfelder M, Nimsky C: Quantification of glioma removal by intraoperative highfield magnetic resonance imaging: an update. Neurosurgery 69:852-863, 2011

14. Kwan P, Arzimanoglou A, Berg AT, Brodie MJ, Allen Hauser W, Mathern G, et al: Definition of drug resistant epilepsy: consensus proposal by the ad hoc Task Force of the ILAE Commission on Therapeutic Strategies. Epilepsia 51:10691077,2010

15. Luyken C, Blümcke I, Fimmers R, Urbach H, Wiestler OD, Schramm J: Supratentorial gangliogliomas: histopathologic grading and tumor recurrence in 184 patients with a median follow-up of 8 years. Cancer 101:146-155, 2004

16. Majores M, von Lehe M, Fassunke J, Schramm J, Becker AJ, Simon M: Tumor recurrence and malignant progression of gangliogliomas. Cancer 113:3355-3363, 2008

17. Olivier A, Bohling WW, Tanriverdi T: Epilepsy and brain tumors, in Techniques in Epilepsy Surgery: The MNI Approach. Cambridge, UK: Cambridge University Press, 2012, pp 216-233

18. Roessler K, Sommer B, Grummich P, Coras R, Kasper BS, Hamer HM, et al: Improved resection in lesional temporal lobe epilepsy surgery using neuronavigation and intraoperative MR imaging: favourable long term surgical and seizure outcome in 88 consecutive cases. Seizure 23:201-207, 2014

19. Schramm J, Aliashkevich AF: Surgery for temporal mediobasal tumors: experience based on a series of 235 patients. Neurosurgery 60:285-295, 2007

20. Senft C, Bink A, Franz K, Vatter H, Gasser T, Seifert V: Intraoperative MRI guidance and extent of resection in glioma surgery: a randomised, controlled trial. Lancet Oncol 12:997-1003, 2011

21. Sommer B, Grummich P, Coras R, Kasper BS, Blumcke I, Hamer HM, et al: Integration of functional neuronavigation and intraoperative MRI in surgery for drug-resistant extratemporal epilepsy close to eloquent brain areas. Neurosurg Focus 34(4):E4, 2013

22. Sommer B, Grummich P, Hamer H, Bluemcke I, Coras R, Buchfelder M, et al: Frameless stereotactic functional neuronavigation combined with intraoperative magnetic resonance imaging as a strategy in highly eloquent located tumors causing epilepsy. Stereotact Funct Neurosurg 92:59-67, 2014

23. Stefan H, Buchfelder M: Presurgical evaluation for epilepsy surgery. Neurol Psych Brain Res 7:109-112, 1999
24. Southwell DG, Garcia PA, Berger MS, Barbaro NM, Chang EF: Long-term seizure control outcomes after resection of gangliogliomas. Neurosurgery 70:1406-1414, 2012

25. Surges R, Elger CE: Reoperation after failed resective epilepsy surgery. Seizure 22:493-501, 2013

26. Thom M, Blümcke I, Aronica E: Long-term epilepsy-associated tumors. Brain Pathol 22:350-379, 2012

27. van Breemen MS, Wilms EB, Vecht CJ: Epilepsy in patients with brain tumours: epidemiology, mechanisms, and management. Lancet Neurol 6:421-430, 2007

28. Walker DG, Talos F, Bromfield EB, Black PM: Intraoperative magnetic resonance for the surgical treatment of lesions producing seizures. J Clin Neurosci 9:515-520, 2002

29. Wallace D, Ruban D, Kanner A, Smith M, Pitelka L, Stein J, et al: Temporal lobe gangliogliomas associated with chronic epilepsy: long-term surgical outcomes. Clin Neurol Neurosurg 115:472-476, 2013

30. Wolf HK, Campos MG, Zentner J, Hufnagel A, Schramm J, Elger CE, et al: Surgical pathology of temporal lobe epilepsy. Experience with 216 cases. J Neuropathol Exp Neurol 52:499-506, 1993

31. Yang I, Chang EF, Han SJ, Barry JJ, Fang S, Tihan T, et al: Early surgical intervention in adult patients with ganglioglioma is associated with improved clinical seizure outcomes. J Clin Neurosci 18:29-33, 2011

32. Zentner J, Hufnagel A, Wolf HK, Ostertun B, Behrens E, Campos MG, et al: Surgical treatment of neoplasms associated with medically intractable epilepsy. Neurosurgery 41:378-387, 1997

\section{Author Contributions}

Conception and design: Sommer, Hamer, Roessler. Acquisition of data: Sommer, Wimmer, Coras, Blumcke, Lorber, Hamer. Analysis and interpretation of data: all authors. Drafting the article: Sommer, Coras, Roessler. Critically revising the article: Sommer, Blumcke, Hamer, Stefan, Buchfelder, Roessler. Reviewed submitted version of manuscript: Sommer, Coras. Approved the final version of the manuscript on behalf of all authors: Sommer. Statistical analysis: Sommer, Wimmer. Study supervision: Roessler.

\section{Supplemental Information \\ Proceedings}

Portions of this work were presented as an oral presentation at the 65th Annual Meeting of the German Neurosurgical Society, Dresden, Germany, May 2014.

\section{Correspondence}

Björn Sommer, Department of Neurosurgery, University Hospital Erlangen, Schwabachanlage 6, 91054 Erlangen, Germany. email: bjoern.sommer@uk-erlangen.de. 